\title{
DIFENIL ÉTERES POLIBROMADOS (PBDES) - NOVOS POLUENTES, ANTIGOS DESAFIOS
}

\author{
Daniele Botaro $^{1}$ \& João Paulo Machado Torres ${ }^{1}$ \\ ${ }^{1}$ Laboratório de Radioisótopos Eduardo Penna Franca, Inst. de Biofísica Carlos Chagas Filho, Universidade Federal do Rio de Janeiro (UFRJ). Ilha do \\ Fundão. Sala 61, subsolo. CEP: 21941-902. Rio de Janeiro, Brasil. \\ E-mails: danibot13@biof.ufrj.br; jptorres@biof.ufrj.br.
}

\section{RESUMO}

Nesse artigo nós revisamos alguns dados recentemente disponíveis sobre difenil éteres polibromados (PBDEs) em amostras ambientais e tecidos humanos em diversas regiões do mundo. Os PBDEs são retardantes de chama adicionados a produtos de consumo para reduzir e evitar a propagação do fogo, como em computadores, equipamentos eletrônicos, carpetes e estofados usados em casas, carros, ônibus, caminhões e aviões. São aditivos retardantes de chama misturados a polímeros, mas não se apresentam quimicamente ligados às estruturas poliméricas dos plásticos ou tecidos o que pode facilitar a separação e lixiviação destes compostos da surperfície dos produtos nos quais são aplicados e contaminar o meio ambiente. O aumento desses poluentes no ambiente tem acentuado a preocupação de cientistas nas últimas décadas. Revisões sobre uso, ocorrência e toxicidade de PBDEs indicam muitas semelhanças entre esses e as bifenilas policloradas (PCBs) e outros poluentes polihalogenados orgânicos persistentes e sugere que os PBDEs podem ser um significante desafio no futuro. As principais conseqüências toxicológicas de altas concentrações de PBDEs em humanos são disrupção de hormônios da tireóide, déficits neurológicos durante o desenvolvimento fetal e até câncer.

Palavras-chave: Difenil éteres polibromados, consumo de peixe, saúde, poluição ambiental.

\section{ABSTRACT}

POLYBROMINATED DIPHENYLETHERS(PBDES)-NEW POLLUTANTS, OLDCHALLENGES. In this paper, we review those data which have recentently became available for polybrominated diphenyl ethers (PBDEs) in environment samples, and human tissues from several world areas. The PBDEs are flame-retardant chemicals that are added to a variety of consumer products to make them difficult to burn like computers, electrical equipments, carpets and upholstery used in household and business furnishings, cars, buses, trucks, and airplanes. PBDEs are additives mixed into polymers and are not chemically bound to the plastic or textiles and therefore may separate or leach from the surface of their product applications into the environment. The presence of these pollutants has become of increasing concern to scientists over the past decade. Review of the use, occurrence, and toxicity of PBDEs indicates many parallels between some PBDEs, PCBs, and other polyhalogenated persistent organic pollutants, and suggests that the PBDEs may be a significant environmental challenge in the future. The toxicological endpoints of high levels of PBDEs in humans are likely to be thyroid hormone disruption, neurodevelopmental deficits and cancer.

Key-words: Polybrominated diphenyl ethers, comsuptiom of fish, health, environment pollution.

\section{INTRODUÇÃO}

Um estudo com concentrações de difenil éteres polibromados (PBDEs) em humanos revelou um recorde alarmante. A média da concentração de PBDEs em um grupo de pessoas residentes em Nova Iorque, Estados Unidos foi de 77,3ng/g de lipídeo, com destaques para os maiores valores de 9630ng/g em um homem de 32 anos de idade e 4060ng/g em uma mulher de 23 anos de idade (Kannan et al. 2005). Resultados de estudos como este, têm sido cada vez mais freqüentes e a preocupação com essa nova classe de poluentes tem aumentado vertiginosamente entre a comunidade científica e os órgãos de saúde do mundo todo.

Difenil éteres polibromados (PBDEs) são substâncias químicas conhecidas como retardantes de chama, adicionadas a plásticos usados em diversos produtos de 
consumo para evitar a propagação do fogo (de Wit 2002). Mais de 175 tipos diferentes de retardantes de chama estão atualmente disponíveis no mercado. Estes estão divididos em diversas classes de compostos, incluindo os halogenados orgânicos (bromados e clorados), compostos de fósforos, nitrogênio e alguns inorgânicos.

Retardantes de chama têm sido adicionados a produtos de consumo como eletrônicos, carpetes e estofados usados em casas, veículos, aviões, entre outros, desde os anos 70 (Mcdonald2002). São aditivos misturados a compostos químicos, como resinas, por exemplo, mas não são quimicamente ligados a esses compostos (de Wit 2002). Atividades como lavagem com ácidos, incineração de componentes de computadores, cabos e fios a céu aberto, deposição de motores e transformadores em lixo comum, podem resultar na descarga de efluentes contendo esses compostos no ambiente (Peng et al. 2007).

\section{CARACTERIZAÇÃO QUÍMICA DOS PBDES}

Os PBDEssãoestruturalmente similaresàsbifenilas policloradas (PCBs), com a mesma nomenclatura e número de congêneres. São 209 congêneres ou diferentes tipos de moléculas, numerados de acordo com a IUPAC (União Internacional de Química Pura e Aplicada; de Wit 2002). Baseados no número de bromo substituintes há 10 grupos homólogos de PBDEs congêneres, onde cada grupo possui um ou mais isômeros. Os mono, di, tri, tetra, penta, hexa, hepta, octa, nona e decabromo congêneres podem existir em 3, 12, 24, 42, 46, 42, 24, 12, 3, e 1 isômeros, respectivamente (ATSDR 2004). A estrutura química geral dos PBDEs é a seguinte:

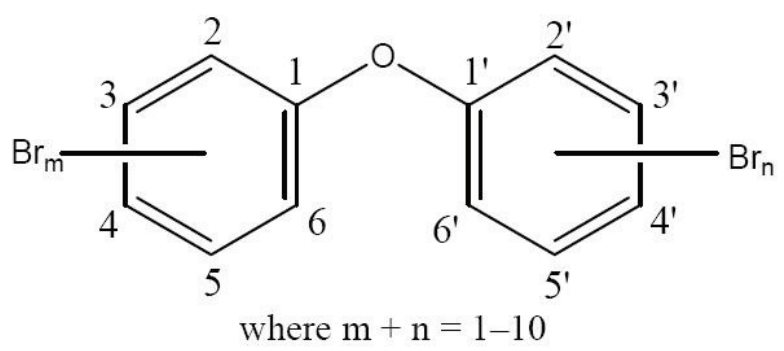

Figura 1. Estrutura química geral dos PBDEs, onde $m+n=1-10$ (Fonte: ATSDR 2004)

Os três PBDEs comerciais mais vendidos e consumidos no mundo são pentabromodifenil éter (pentaBDE) com cinco átomos de bromo, octabromodifenil éter (octaBDE) com oito átomos de bromo e decabromodipenil éter (decaBDE) com 10 átomos de bromo em sua estrutura molecular (McPherson et al. 2004). As misturas comerciais decaBDE e octaBDE são sólidas e transparentes, enquanto que a de pentaBDE é líquida. Na tabela I podemos ver diversas propriedades químicas e físicas das três principais misturas comercias de PBDEs.

O penta BDE é usado predominantemente (9598\%) como um aditivo retardante de chama pela indústria de móveis. É usado quase que exclusivamente como retardante de chama em espumas de poliuretano (FPUF), que são usadas em colchões e no estofamento de sofás e bancos em geral. A mistura comercial pentaBDE é usada nessas espumas como um aditivo misturado com ésteres fosfatos aromáticos (por exemplo 75\% pentaBDE e 25\% ésteres fosfatos aromáticos). Sucata e restos dessas indústrias têm sido usados para confecção de carpetes e, portanto, estes possuem de 3-5\% de PBDEs. Entretanto, nem toda espuma consumida no mundo hoje apresenta PBDEs. Aproximadamente 7,5\% de cerca de 1 bilhão de quilos de espuma produzidos anualmente só nos Estados Unidos, possuem pentaBDE (ENVIRON 2003). Esse composto ainda pode ser encontrado em adesivos, tecido, circuitos de impressoras, produtos de borracha, epóxi, laminados, roupas, poliésteres insaturados e polivinil (PVC) (Great Lakes Chemical Corporation 2004).

OctaBDE é usado em plásticos para equipamentos, principalmente para retardar chama em acrilonitrilabutadieno-styrene (ABS) termopolímeros usados em gabinetes de computadores e monitores (ENVIRON 2003). Na União Européia, cerca de $95 \%$ do total de octaBDE é vendido em eletrônicos e indústrias que usam plástico ABS. Nesses plásticos ABS, estão presentes aproximadamente $12-18 \%$ peso do produto final em retardantes de chama. O octaBDE é sempre usado em conjunto com trióxido de antimônio. Essa mistura contendo oito átomos de bromo em sua molécula também pode ser usada em polietileno de alto impacto (HIPS), polímeros de poliamidapolicarbonatos, resinas de fenolformaldeído (EU 2003), nylon, termoplásticos e adesivos (Great Lakes Chemical Corporation 2004).

O composto comercial decabromodifenil éter compõe cerca de $82 \%$ de todos os produtos manufaturados globalmente. A indústria da informação 
Tabela I. Propriedades químicas e físicas de três misturas comercias de Difenil Éteres Polibromados - PBDEs (Adaptada de ATSDR 2004).

\begin{tabular}{|c|c|c|c|}
\hline Propriedade & PentaBDE & OctaBDE & DecaBDE \\
\hline Peso molecular & Mistura & Mistura & 959,22 \\
\hline Cor & Claro, de âmbar a amarelo & Transparente & Transparente \\
\hline Estado físico & Líquido altamente viscoso & Pó & Pó \\
\hline Ponto de Fusão & $-7 \mathrm{a}-3^{\circ} \mathrm{C}$ (comercial) & $\begin{array}{c}85-89^{\circ} \mathrm{C} \text { (comercial); } 200^{\circ} \mathrm{C} \\
(167-257)\end{array}$ & $290-306^{\circ} \mathrm{C}$ \\
\hline Ponto de Ebulição & $\begin{array}{c}>300^{\circ} \mathrm{C} \text { (decomposição inicia } \\
\text { após } 200^{\circ} \mathrm{C} \text { ) }\end{array}$ & $\begin{array}{c}\text { Decomposição acima de } \\
330^{\circ} \mathrm{C} \text { (comercial) }\end{array}$ & $\begin{array}{l}\text { Decomposição acima de } \\
320\left(>400 \text { e } 42^{\circ} \mathrm{C}\right)\end{array}$ \\
\hline Densidade (g/mL) & 2,28 а $25^{\circ} \mathrm{C} ; 2,25-2,28$ & 2,76; 2,8 (comercial) & 3,$0 ; 3,25$ \\
\hline Solubilidade & $2,4 \mu \mathrm{g} / \mathrm{L}$ (pentaBDE) & & $<0,1 \mu \mathrm{g} / \mathrm{Lg}$ \\
\hline Água & $10,9 \mu \mathrm{g} / \mathrm{L}($ tetraBDE$)$ & $1,98 \mu \mathrm{g} / \mathrm{L}$ (heptaBDE) & \\
\hline Solventes orgânicos(s) & $\begin{array}{c}\text { 10g/kg metanol; miscível em } \\
\text { tolueno }\end{array}$ & $\begin{array}{c}\text { Acetona }(20 \mathrm{~g} / \mathrm{L}) \\
\text { Benzeno }(200 \mathrm{~g} / \mathrm{L}) \\
\text { Metanol }(2 \mathrm{~g} / \mathrm{L}) \text { a } 25^{\circ} \mathrm{C}\end{array}$ & $\begin{array}{c}\text { Acetona }(0,05 \%) \text {, benzeno } \\
(0,48 \%), \\
\text { Tolueno }(0,2 \%)\end{array}$ \\
\hline $\begin{array}{l}\text { Coeficientes de partição } \\
\text { Log Kow } \\
\text { Log Koc }\end{array}$ & $\begin{array}{l}6,64-6,97 \\
4,89-5,10\end{array}$ & $\begin{array}{c}\text { 6,29 (comercial) } \\
5,92-6,22\end{array}$ & $\begin{array}{c}6,265 \\
6,80\end{array}$ \\
\hline Pressão de vapor & $\begin{array}{c}2,2 \times 10^{-7}-5,5 \times 10^{-7} \mathrm{~mm} \mathrm{Hg} \text { a } 25 \\
{ }^{\circ} \mathrm{C} ; 3,5 \times 10^{-7} \mathrm{~mm} \mathrm{Hg}\end{array}$ & $\begin{array}{c}9,0 \times 10^{-10}-1,7 \times 10^{-9} \mathrm{~mm} \\
\mathrm{Hg} \text { a } 25^{\circ} \mathrm{C} ; 4,9 \times 10^{-8} \\
\text { mm Hg a } 21^{\circ} \mathrm{C}\end{array}$ & $\begin{array}{l}3,2 \times 10^{-8} \mathrm{~mm} \mathrm{Hg} \\
3,47 \times 10^{-8} \mathrm{~mm} \mathrm{Hg}\end{array}$ \\
\hline $\begin{array}{l}\text { Constante de Henry's Law } \\
\left(\mathrm{atm}-\mathrm{m}^{3} / \mathrm{mol}\right)\end{array}$ & $\begin{array}{c}1.2 \times 10^{-5} ; 1.2 \times 10^{-6} \\
3.5 \times 10^{-6}\end{array}$ & $\begin{array}{c}7,5 \times 10^{-8} ; 2,6 \times 10^{-7} \\
1,62 \times 10^{-6} ; 1,93 \times 10^{-8}\end{array}$ & $1,2 \times 10^{-8} ; 4,4 \times 10^{-8}$ \\
\hline
\end{tabular}

Fonte: Adaptada de ATSDR 2004.

indica que os decaBDE representam de $10-15 \%$ do peso de polímeros e é sempre usado em conjunto com trióxido de antimônio. A maior aplicação dessa mistura é em HIPS, os quais são usados em televisores e gabinetes de computadores. É usado também em diversos polímeros cujo destino final é a confecção de componentes eletrônicos (computadores, conectores, caixas elétricas, fios e cabos, etc.), polipropileno, copolímeros acetatos (etileno vinil acetato - EVA), termoplásticos e resinas poliésteres (EU 2002). Nos Estados Unidos (EUA), móveis estofados devem seguir um padrão de normas de flamabilidade e podem usar cerca de $5 \mathrm{mg} / \mathrm{m}^{2}$ de decaBDE (BFRIP 2002). Além dessas aplicações, os decaBDEs são amplamente utilizados em tecidos para móveis, carros e aeronaves, estimando-se que cerca de 10 a $20 \%$ de todo o decaBDE consumido hoje nos EUA, seja para essa finalidade (Lowell 2005).

\section{PBDEs NO AMBIENTE}

Em todo o mundo, a demanda por decaBDE foi de 56100 toneladas em 2001; 65677 t em 2002; e 56419 t em 2003 (BSEF 2006). Por não ser quimicamente ligado aos compostos nos quais são adicionados, os decaBDE são amplamente encontrados no ambiente (Lowell 2005). Potenciais produtos de degradação do decaBDE incluem os nonaBDEs 206-208, o octaBDE 203, o hexaBDEs 153, 154 e 183, o pentaBDE 99 e o tetraBDE 47. Os PBDEs 47 e 99 também são encontrados na mistura comercial de pentaBDE e são os mais encontrados em estudos ambientais. Mais recentemente, os congêneres 153, 154 e 183 também têm aumentado suas concentrações em amostras ambientais e em tecidos humanos e animais.

Os PBDEs são lançados no ambiente através do ar, água e solo, durante a sua utilização e por queima de plásticos que continham esse poluente. Em 1973, em Michigan nos Estados Unidos, animais foram acidentalmente alimentados com rações contendo PBDEs, ocorrendo uma grande contaminação da cadeia alimentar, atingindo também pessoas que ingeriram carne, leite e ovos de animais daquele local (Webster 2004).

A preocupação com esses poluentes tem aumentado a medida que maiores concentrações dos mesmos tem sido identificadas em estudos no mundo todo e começam a ser associados a danos á saúde 
humana. Estudos com uso, ocorrência e toxicidade de PBDEs tem indicado muitas semelhanças com as bifenilas policloradas (PCBs) e outros poluentes orgânicos persistentes (POPs), o que sugere que esses novos poluentes sejam um desafio ambiental no futuro (Ikonomou et al. 2005). Uma das razões que explica esse aumento nos níveis de PBDEs no ambiente é o consumo cada vez maior de produtos eletrônicos contendo esses poluentes o que gera o chamado lixo tecnológico. O ATSDR de $2004 \mathrm{fez}$ um cálculo baseado no número de computadores obsoletos contendo retardantes de chama entre 1997 e 2004 e chegou ao total de 315 milhões. Baseado no peso de um monitor de $14 \mathrm{~kg}$, isso resultaria em 160 milhões de $\mathrm{kg}$ de retardantes de chama que foram lançados no ambiente. Embora os PBDEs sejam apenas uma fração desse total, ainda assim o número é preocupante (ATSDR 2004). Além dos equipamentos eletrônicos estudos revelam que são encontrados maiores níveis de PBDEs em carros do que em casas e que em carros novos esses níveis são mais altos do que em carros antigos. Além dos carros, altos níveis desses poluentes foram detectados no ar de escritórios e de locais públicos fechados no Reino Unido (Hazrati \& Harrad 2006).

Uma vez no ambiente, esses compostos podem estar passíveisdedegradaçãoegerarmetabólitosquetambém são tóxicos ao meio ambiente e conseqüentemente a vida animal. Os PBDEs são mais bioacumulativos e possuem maior potencial de transporte na atmosfera, comparado as PCBs (Ikonomou et al. 2005), além de serem aparentemente mais susceptíveis a degradação ambiental, pois o bromo é mais reativo quimicamente do que o cloro, podendo se ligar a uma gama maior de outros compostos.

As vias de contaminação de PBDEs por humanos são ar, água, solo e sedimento, mas principalmente através da ingestão de alimentos, particularmente os que contêm alto teor de gordura. A incineração de lixo contendo PBDEs também pode ser uma fonte de contato, pois esse processo pode gerar a formação de PBDFs (furanos) e PBDDs (dioxinas).

Os caminhos pelos quais os PBDEs podem entrar no organismo depende de sua estrutura química. Os congêneres com maior número de bromos, particularmente o decaBDE possuem formas bastante exclusivas de entrada no organismo, diferente dos compostos menores de PBDEs. O decaBDE inteiro é pobremente absorvido, rapidamente eliminado e não bioacumula (Hooper \& McDonald 2000). Ao contrário, congêneres com menor número de bromos, como o tri, tetra, penta e hexaBDEs, são quase que completamente absorvidos, lentamente eliminados e altamente bioacumulativos. Uma importante observação é que os decaBDE, quando expostos a luz solar, são convertidos em moléculas menores, representando assim um risco maior de contaminação (Mcdonald 2002).

Congêneres menores de PBDEs podem entrar no corpo humano através do pulmão, pele e estômago e em seguida passar para a corrente sanguínea. Uma vez no organismo, os congêneres maiores podem se quebrar em metabólitos e serem eliminados nas fezes e uma pequena parte na urina. Congêneres menores, geralmente tetra, penta e hexaBDE, podem permanecer no corpo acumulados no tecido adiposo por até 30 anos, por serem compostos altamente lipofílicos (Li 2005). Esses congêneres menores, também podem se concentrar na gordura do leite materno e serem transmitidos aos recém nascidos no momento da amamentação, além de também serem transmitidos pela placenta (ATSDR 2004). Devido à grande preocupação dos danos desses compostos à saúde humana, estudos têm sido realizados em diversas matrizes ambientais e humanas a fim de diagnosticar a ocorrência desses poluentes e tentar evitar a sua dissipação.

Os PBDEs possuem baixa pressão de vapor e no ar apresentam-se em fase particulada, sendo removidos da atmosfera pela deposição seca e úmida (Schmid $e t$ al. 2007). Entretanto, alguns congêneres da mistura de pentaBDE, como 2,2',4,4'-tetrabromodifenil éter (BDE 47) e 2,2',4,4',5-pentabromodifenil éter (BDE 99), têm sido encontrados em regiões do Ártico. Não há uma explicação de como esses congêneres foram transportados das possíveis áreas de emissão até esses pontos distantes. Entretanto, provavelmente eles podem ter sido transportados através de partículas de poeira. Na água os PBDEs adsorvem-se fortemente com sólidos em suspensão e sedimento e bioconcentram em organismos aquáticos, o que reduz a chance desses compostos terem sido transportados através de volatilização.

Em um estudo do ar entorno dos Grandes Lagos, nos Estados Unidos, as concentrações atmosféricas de PBDEs foram altamente correlacionadas com as de 
PCBs (Hites et al. 2004). Estudos em Chicago (EUA) mostraram que havia $5-10$ vezes mais PBDE no ar próximo a área urbana do que na área rural (Strandberg et al. 2001) o que reforça a teoria de outros estudos que comprovam que áreas urbanas apresentam maiores concentrações de PBDEs do que área rurais (Harner et al. 2006, Harrad \& Hunter 2006).

Outra constatação é que em ambientes fechados como escritórios, podemos encontrar altas concentrações de PBDEs. Em estudo realizado com três grupos de trabalhadores (1- trabalhadores de uma indústria desmontadora de computadores, 2trabalhadores de um escritório e 3 - grupo controle), Sjödin et al. (1999), observaram que as concentrações médias de PBDEs no soro sanguíneo (soma de congêneres) foram maiores nos trabalhadores da indústria desmontadora de computadores (26ng/g lipídeo); seguido pelos trabalhadores de escritório (4,1ng/g lipídeo) e do grupo controle (3,3ng/g lipídeo). O padrão de congêneres encontrados no grupo controle e nos trabalhadores de escritório foi o mesmo, com maiores níveis de BDE-47. Para os trabalhadores da indústria desmontadora de computadores, as médias das concentrações de BDE183, BDE-153, BDE-154, BDE-47 e BDE-209 foram 7,8; 4,5; 1,2; 2,9 e 4,8 ng/g lipídeo, respectivamente. Hagmar et al. (2000) observaram as que concentrações de BDE-153, BDE-183 e BDE-209 no soro sanguíneo de 19 técnicos de computadores foi cinco vezes maior do que a observada por Sjodin et al. (1999) para os trabalhadores do grupo controle e os de escritório.

Harner etal. (2006) calcularam a máxima exposição diária a PBDEs de uma pessoa pela respiração em casa. Os níveis médios encontrados corresponderam a baixa inalação de 1,9ng/dia para mulheres e 2,0ng/ dia para homens, visto que estes apresentam maior taxa de inalação do que as mulheres. Porém, os níveis variam de acordo com o nível de poluição das casas, podendo chegar a 66ng/dia de PBDEs para mulheres e 71ng/dia para homens.

Os PBDEs são fortemente adsorvidos pelo solo e sedimento e a adsorção aumenta de acordo com o número de átomos de bromo e o número de carbonos orgânicos contidos no solo ou sedimento. Como resultado, a maioria dos PBDEs tem baixa ou nenhuma mobilidade no solo, não sendo esperado que ocorra lixiviação dos mesmos. Esses poluentes chegam ao solo através da deposição de lixo doméstico e industrial, além da deposição de restos da indústria de PBDEs que é geralmente realizada em aterros (EPA 1995). Microorganismos no solo e sedimento podem quebrar PBDEs em congêneres com menor número de bromos, através da degradação anaeróbica (Robrock et al. 2005). Dentre as formas encontradas no ambiente e tecidos animais, os BDE-47 e BDE-99 têm demonstrado alta toxicidade e são resultados da degradação de pentaBDE (He et al. 2006).

Hale et al. (2002) observaram a concentração em solos e sedimentos coletados próximos a uma fábrica de poliuretano (próximo ao Rio Dan, Virginia, EUA). Os congêneres dominantes nessas amostras foram 2,2',4,4'-tetraBDE (BDE 47), 2,2',4,4',5-pentaBDE (BDE-99) e 2,2',4,4',6-pentaBDE (BDE-100), e as concentrações totais variaram de $<1$ to $132 \mathrm{ng} / \mathrm{g}$ de peso seco. Em 1995, amostras de sedimento foram coletadas próximas a uma indústria de plásticos (rio abaixo e rio acima) que usavam retardantes de chama na Suécia e foram encontradas concentrações de tetraBDEs de 50ng/g peso seco e pentaBDEs de 2300ng/g peso seco (soma de três congêneres). As maiores concentrações foram detectadas rio abaixo, indicando que a fábrica é uma potencial fonte de contaminação (Sellström et al. 1998).

Variações em fontes locais, combinadas com transporte atmosférico podem explicar diferenças no perfil de congêneres de PBDEs em diferentes lagos. Efluentes industriais e urbanos também são fontes significantes de PBDEs para águas superficiais e sedimentos.

Os PBDEs estão presentes em alimentos consumidos na nossa dieta, com mais altas concentrações em produtos de origem animal do que em frutas e vegetais (Mueller et al. 2006). Schecter et al. (2006) analisando níveis de PBDEs em 62 amostras de peixes, carne e produtos do leite e derivados nos EUA, encontrou os maiores níveis em peixes. O BDE-47 foi o congênere dominante seguido pelo BDE-99, BDE100 e decaBDE. A carne teve maiores concentrações de PBDEs do que o leite e seus derivados, sendo também encontrado nesses produtos, predominantemente o congênere 47.

Ospeixes sãoparticularmente mais ricos em $\mathrm{PBDEs}$ como resultado da bioacumulação e bioconcentração em ambientes aquáticos, como constatado na Europa e América do Norte (Hamilton \& Fisher 2006, Knutsen et al. 2006, Tlustos et al. 2005a, 2005b). Gordura 
de peixe apresenta os maiores níveis de PBDEs, principalmente no salmão (Domingo et al. 2006) e peixes provenientes de criação comercial apresentam maiores níveis de PBDEs do que peixes em ambiente natural (Tlustos et al. 2005a, Hamilton \& Fisher 2006). Estudos mostram que as concentrações de PBDEs em salmão criado pode diminuir quando se faz substituição de óleo de peixe por óleos vegetais nas rações (Bethune et al. 2006).

O salmão criado, coletado em dois diferentes locais do Canadá apresentou maiores níveis de BDE47 (690 e 2600ng/g peso úmido) seguido pelo BDE99 e BDE-100, respectivamente onde a soma total dos congêneres foi de 1188 e 4147ng/g peso úmido, respectivamente para os dois locais de coleta. Em outro estudo, salmão selvagem foi coletado de quatro locais diferentes e estes apresentaram concentrações 10 vezes menores de PBDEs (concentrações totais de 38,7 a 485,2ng/g peso úmido e de BDE-47de 29 a 280ng/g peso úmido), do que de peixes provenientes de criação (Easton et al. 2002).

Um estudo realizado na Alemanha mostrou que os níveis de PBDEs em óleo de peixe eram duas vezes maiores do que os encontrados no tecido, com base em lipídeos. Além disso, o decaBDE, apesar de ser o congênere menos encontrado no tecido desses animais, em um dos indivíduos analisados apresentou a concentração de 4800ng/g lipídeo (Hermann et al. 2005).

DecaBDE e octaBDE não são bioconcentrados em peixes. Os fatores de bioconcentração (BCFs) para essas misturas comercias são geralmente menores que 50 (Hardy 2002). Um simples estudo feito com uma mistura de PBDEs (entre eles, hexaBDE e decaBDE) indicou uma baixa bioconcentração em carpas (Cyprinus carpio) cujo fator de bioconcentração foi quatro depois de oito semanas de exposição.

Para o pentaBDE os dados são diferentes. Estudos comprovam que há alta bioconcentração desses compostos em organismos aquáticos com BCFs de até 14000 (Hardy 2002). Congêneres que compõem a mistura comercial de pentaBDE bioconcentram de formas diferentes. Por exemplo, aproximadamente 50-70\% dos PBDEs detectados em peixes eram de um único isômero BDE-47, seguido pelo BDE-99 e pelo BDE-100. Em um estudo de laboratório em "blue mussels" do mar Báltico (Mytilusedulis L), o BCFs de absorção da água foi de 1300000 para BDE-
47, 1400000 para BDE-99 e 1300000 para BDE-153 (Gustafsson et al. 1999). Em diversos locais ao longo da costa do estuário de Schelde na Holanda, os BCFs para "blue mussels" foram de $1 \times 10^{9}$ para BDE-99 e

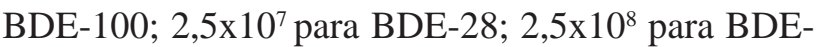

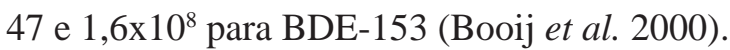

A bioacumulação de PBDEs na cadeia alimentar é inversamente proporcional ao grau de brominação dos compostos. Congêneres com alto número de bromos em sua molécula (decaBDE) são raramente detectados na biota e isso é resultado de sua baixa solubilidade, altos valores de $\log$ Kow e alto grau de adsorção no solo e sedimento (Hardy 2002). Em contraste, tetraBDE, pentaBDE e hexaBDE homólogos são freqüentemente encontrados na biota o que se deve a grande solubilidade em água e relativos baixos valores de $\mathrm{K}_{\mathrm{ow}}$ (Hale et al. 2003).

\section{TOXICIDADE E VIAS CRÍTICAS PARA OS SERES HUMANOS}

Em leite, sangue e tecidos tem se observado um aumento exponencial nos níveis de PBDEs com um fator de quase 100 durante os últimos 30 anos. São preocupantes os níveis de PBDEs encontrados na população do mundo todo, com destaque aos EUA, que comparado com a Europa apresentam valores alarmantes. As atuais concentrações de PBDE em europeus são de 2ng/g lipídeo e nos EUA esses níveis já chegam a 35ng/g lipídeo (Hites et al. 2004). Isso pode ser explicado pelo maior uso dessas misturas comerciais pelos americanos, quase que 50 vezes mais do que na Europa (BSEF 2006).

Recente atenção tem sido dada às conseqüências da deposição inadequada de produtos contendo PBDEs, como por exemplo, o descarte de computadores e equipamentos eletrônicos. Altos níveis de PBDEs são encontrados próximos a fábricas que fazem reciclagem desse material, também chamado de "ewaste" (Cai \& Jiang 2005) e o padrão de congêneres depende diretamente do produto que é manuseado nessas fábricas (Wang et al. 2005). PBDEs foram analisados em ewaste, autoshredder waste e outros resíduos sólidos (carpetes, móveis) na Califórnia onde, autoshredder waste teve como congênere dominante o decaBDE, com a maior concentração total em relação aos produtos analisados. Os outros resíduos sólidos tiveram como congêneres 
dominantes penta e octaPBDEs (Petreas et al. 2005, Petreas \& Oros 2006).

Thuresson et al. (2006), em estudo realizado com trabalhadores que reciclam produtos eletrônicos, observaram que os trabalhadores que ficavam em uma área mais limpa e controlada da fábrica apresentavam menores níveis de PBDEs no sangue. $\mathrm{O}$ ar interno da fábrica continha altos níveis de BDE-47 e BDE-153, os quais também foram encontrados em altas concentrações no sangue dos trabalhadores. Esses congêneres de PBDE possuem longo tempo de meia-vida, de 1,8 anos para BDE-47 e 6,5 para BDE-153. Devido a esse grande tempo de meia-vida, o congênere BDE153 está sendo encontrado em altas concentrações no sangue de populações da Holanda (Weiss et al. 2006), Ilhas Faroe (Fangstrom et al. 2005) e Suécia (Thuresson et al. 2005a). Estimativas de concentrações de PBDEs no sangue de trabalhadores de indústrias desmontadoras de produtos eletrônicos, antes e depois de um período de férias de 28 dias, indicam que os maiores congêneres têm tempos de meia-vida mais curtos do que congêneres menores (Sjödin et al. 1999).

Assim, o padrão de congêneres encontrados em humanos depende diretamente do padrão de congêneres ao qual a pessoa foi exposta (Hooper \& McDonald 2000). São três os congêneres padrões encontrados em tecidos humanos: BDE-47, BDE-99 e BDE-153. Esses foram detectados em amostras de tecido adiposos na população da Suécia com níveis variando de 0,3 a 98,2ng/g lipídeo (Haglund et al. 1997, Hardell et al. 1998).

A acumulação desses congêneres menores de PBDEs é principalmente por causa de sua fácil absorção, metabolismo e eliminação, se comparados aos congêneres maiores como o decaDBE. Soro sanguíneo, tecido adiposo e leite materno são indicadores da exposição humana aos PBDEs, onde também há predominância dos congêneres BDE-47, BDE-99 e BDE-153. E os estudos mostram que essas concentrações estão aumentando com o passar dos anos. Em estudo realizado com concentrações de PBDEs em leite materno de mães da Suécia de 1972 a 1997, Norén \& Meironyté (2000) observaram que a concentração desses poluentes dobra a cada cinco anos. Em 1997 as amostram continham concentrações de PBDEs (soma de 8 congêneres) de 4ppb com base em lipídeos enquanto que em 1972 esses níveis não chegavam a $0,07 \mathrm{ppb}$.
PBDEs também têm sido identificados na placenta de mulheres grávidas, e isso é um fato preocupante, pois esses poluentes podem ser transmitidos ao feto através da placenta. Strandman et al. (2000), observaram níveis de 1,00 a 4,40 ppb com base em lipídeos em amostras de placentas.

Poucas são as definições sobre os efeitos dos PBDEs em humanos, pois a maioria das informações existentes são baseadas em estudos com animais de laboratório. A similaridade química com as bifenilas policloradas (PCBs) também possibilita extrapolar dados para esses poluentes, como este estudo com crianças que após um longo tempo de exposição a PCBs, mostraram que esses poluentes podem causar danos a inteligência e retardo de aprendizagem (McPherson et al. 2004). Estudos comprovam em animais testados e nas análises de sangue e urina das pessoas contaminadas no episódio de Michigan, que a exposição a altas concentrações de PBDEs, causa danos a saúde, como náuseas, dores abdominais, perda de apetite, problemas de pele, perda de cabelo, fadiga e disfunções no sistema nervoso, fígados, rins, glândula tireóide e sistema imune (Nierenberg \& Garcés 2004).

Há três mecanismos possíveis pelos quais os PBDEs (assim como as PCBs não-coplanares) podem afetar o desenvolvimento do cérebro na fase fetal: disrupção dos hormônios tireoidianos, disrupção de mensageiros secundários e alterações no sistema neurotransmissor (Mcdonald 2002).

Todos os congêneres de PBDES podem agir como disruptores do balanço dos hormônios da tireóide. A estrutura química dos PBDEs lembra a desses hormônios 3; 30; 5-triiodotironina (T3) e 3; 30; 5; 50tetraiodotironine(T4). Oshormôniotireoidianos, assim como os metabólitos dos PBDEs, são hidroxilados e halogenados difenil éteres. Recentes estudos in vitro têm mostrado que metabólitos hidroxilados de PBDEs ligam-se com alta afinidade a proteína transportadora dos hormônios tireoidianos (como a transtirretina, por exemplo) (Meerts et al. 2000) além de se ligarem aos receptores TR-a1 e TR-b desses hormônios (Marsh et al. 1998).

A disrupção de hormônios da tireóide é associada com muitos problemas de saúde, incluindo bócio, doenças neoplásicas da tireóide e toxicidades neurológicas e de desenvolvimento, pois sabe-se que o desenvolvimento do cérebro é sensível a disrupção 
da tireóide (Morreale de Escobar et al. 2000). Por exemplo, recentes estudos mostram que decréscimos relativamente baixos de $\mathrm{T} 4$ e outros indicadores de alterações na tireóide podem ter um impacto negativo na inteligência e na habilidade psicomotora de crianças (McDonald 2002).

Em estudo realizado com trabalhadores de uma indústria onde a fonte de PBDE era só de decaBDE, Bahn et al. (1980), observaram que quatro dos 35 trabalhadores amostrados, manifestaram hipotireoidismo. DecaBDE produziu estatisticamente um significante aumento na incidência de hiperplasia da tireóide e uma maior incidência de tumores nessa glândula em ratos machos e fêmeas (NTP 1986). Concentrações de background de PBDEs em tecidos humanos mostraram que esses poluentes estão presentes em maiores concentrações do que dioxinas e PCBs, mas que os níveis de T4 são reduzidos quando há presença concomitante de PBDEs e PCBs (Aroclor 1254) ou parafinas cloradas (Hallgren \& Darnerud 1998).

Os PBDEs também podem interferir nos sistemas de hormônios reprodutivos, incluindo as rotas de estrógenos, andrógenos e progesterona (Nakari \& Pessala 2005, Hamers et al. 2006). Os níveis de PBDEs em leite materno foram associados com diversos resultados em mães e recém nascidos. Peso e comprimento ao nascer e tamanho da circunferência da cabeça foram medidos em recém nascidos e a relação entre os níveis de 12 congêneres de PBDEs no leite foi determinada, indicando que os níveis mais altos de decaBDE eram os do leite das mães de crianças com menores peso e comprimento ao nascer e tamanho da circunferência da cabeça. Nas mães, o decaBDE foi associado com a diminuição do ciclo menstrual e da duração do sangramento, onde os congêneres BDE-100 e BDE-153 foram os principais associados a esse efeitos (Chao et al. 2006).

A toxicidade do neurodesenvolvimento fetal tem sido estudada para BDE-47 e PBDE-99, por serem os mais encontrados em tecidos humanos. Foram administrados 0,7mg de BDE-47 e 10,5mg de BDE99 em ratos no $10^{\circ}$ dia do pósnatal e os resultados foram alterações permanentes no comportamento motor o qual piorou com a idade. A exposição neonatal a BDE-99 também reduziu a capacidade de aprendizagem e memória em ratos adultos. Efeitos similares foram observados em ratos adultos expostos a orto-PCBs e PCBs não-coplanares. Misturas comerciais de penta, octa e decaBDEs aumentaram mortalidade embrionária e atrasaram a formação do esqueleto em ratos (WHO 1994).

A neurotoxicidade em estudos com roedores pode ser o resultado de alterações no sistema neurotransmissor. Viberg et al. (2002), observaram que respostas neurocomportamentais seguidas de exposição posnatal a PBDEs foram consistentes com alterações ao sistema colinérgico. Eriksson et al. (2001), observou que a exposição a BDE-99 e BDE-47 pode causar aberrações permanentes no comportamento. Esse efeito e a capacidade de habituação parecem ser pioradas com o passar do tempo. Além disso, o BDE99 também afeta a aprendizagem e a memória dos animais, como visto em ratos que foram expostos a $12 \mathrm{mg} / \mathrm{kg}$ de peso corporal de BDE-99 e estes apresentaram maior dificuldade de memorizar um labirinto do que os animais do grupo controle.

São limitados os dados a respeito da ocorrência de câncer em humanos devido a exposição a PBDES. Um estudo realizado com pacientes de um hospital na Suécia, mostrou que há associação entre níveis de PBDEs (BDE-47) no tecido adiposo e o risco de nonHodgkin linfoma (NHL) (Hardell et al. 1998).

\section{PERSPECTIVAS}

Em 27 de janeiro de 2003 o Parlamento Europeu e o Council on Waste Electrical Equipment (WEEE) aprovaram uma lei (Directive 2002/95/EC) que entre outras coisas, exigiu que os 16 membros da União Européia fabricassem equipamentos eletrônicos isentos de PBDEs, chumbo, mercúrio, cádmio, cromo hexavalente e PBBs a partir de $1^{\circ}$ julho de 2006 (Kim et al. 2006). Nos Estados Unidos, a regulamentação banirá produtos contendo penta e octaBDE na Califórnia a partir de 2008 e a Great Lakes Chemical, uma das maiores indústrias de misturas comerciais de PBDEs encerrou a produção desses dois PBDEs no fim de 2004 (Hites et al. 2004).

Há evidências de que as indústrias estão progressivamente reduzindo o uso desses poluentes em seus produtos e fazendo a substituição desses por outros retardantes de chama. A Apple (R), por exemplo, está substituindo o plástico usado nos laptops por novos materiais que não necessitam de retardantes de chama. A Toshiba (R) agora usa um 
plástico retardante de chama com poliuretano para gabinetes, monitores e televisões. A NEC (R) tem um novo plástico baseado em fontes vegetais que não necessita de retardantes de chama brominados ou fosforado (McPherson et al. 2004).

Dados alarmantes de altas concentrações de PBDEs em pessoas no mundo todo têm levantado discussões a respeito da exposição humana a esses poluentes. O estudo de Hites et al. (2004) com salmão criado e salmão selvagem, serviu como um alerta para o consumo de peixes e criou um dilema. De um lado nutricionistas impulsionando o consumo de peixe com altos níveis de Ômega-3 e de outro as observações de que cada vez mais poluentes, incluindo os PBDEs são detectados na gordura desses animais. O desenvolvimento de rações formuladas com ingredientes alternativos e menores níveis de poluentes pode ser uma solução para amenizar esse problema (Law et al. 2006).

Alguns especialistas recomendam ações que podem auxiliar na redução da emissão e na exposição humana aos PBDEs. Entre elas, uma efetiva ação de estados e governos para o fim da produção de PBDEs assim como a substituição dos mesmos por outros retardantes de chama. Além disso, aumentar a reciclagem correta de produtos eletrônicos e criar políticas reguladoras para o uso de novos compostos que podem ocasionar danos ao ambiente (McPherson et al. 2004). Um contínuo monitoramento também é recomendado para diminuir o risco de contaminação do ambiente por esses poluentes e cada vez mais estudos são necessários.

AGRADECIMENTOS: Ao CNPq pela bolsa de doutorado concedida e a CAPES/DAAD pelo projeto aprovado de Cooperação Internacional Brasil-Alemanha (doutorado sanduíche).

\section{REFERÊNCIAS}

ATSDR (AGENCY FOR TOXIC SUBSTANCES AND DISEASE REGISTRY). 2004. Toxicological Profile for Polybrominated Biphenyls and Polybrominated Diphenyl Ethers. U.S. Department Of Health And Human Services. Division of Toxicology/Toxicology Information Branch.

BAHN, A.K.; MILLS, J.L.; SYNDER, P.J.; GANN, P.H.; HOUTEN, L.; BIALIK, O.; HOLLMANN, L. \& UTIGER, R.D. 1980. Hypothyroidism in workers exposed to polybrominated biphenyls. The New England Journal of Medicine, 302(1): 31-33.
BETHUNE, C.; SEIERSTAD, S.L.; SELJEFLOT, I.; JOHANSEN, O.; ARNESEN, H.; MELTZER, H.M.; ROSENLUND, G.; FRØYLAND, L. \& LUNDEBYE, A.-K. 2006. Dietary intake of differently fed salmon: a preliminary study on contaminants. European Journal of Clinical Investigation, 36: 193-201.

BOOIJ, K.; ZEGERS, B.N. \& BOON, J.P. 2000. Levels of some polybrominated diphenyl ether (PBDE) flame retardants along the Dutch coast as derived from their accumulation in SPMDs and blue mussels (Mytilus edulis). Organohalogen Compounds, 47: 89-92.

BFRIP (BROMINATED FLAME RETARDANT INDUSTRY PANEL). 2002. Decabromodiphenyl ether (a.k.a. decabromodiphenyl oxide, DBDPO). Voluntary children's chemical evaluation program (VCCEP). Data Summary. Arlington.

BSEF (BROMINE SCIENCE AND ENVIRONMENTAL FORUM). 2006. Fact sheet: brominated flame retardant decaBDE. Bromine Science and Environmental Forum, www. bsef.com, acesso em 12/03/2007.

CAI, Z. \& JIANG, G. 2005. Levels of polybrominated diphenyl ethers in soils from an e-waste recycling site. Organohalogen Compounds, 67: 548-550.

CHAO, H.-R.; WANG, S.-L. \& PÄPKE, O. 2006. Association between birth outcome and polybrominated diphenyl ether levels in breast milk from central Taiwan. Pp. 203. In: 26th International Symposium on Halogenated Persistent Organic Pollutants.

de WIT, C.A. 2002. An overview of brominated flame retardants in the environment. Chemosphere, 46(5):583-624.

DOMINGO, J.L.; BOCIO, A.; FALCÓ, G. \& LLOBET, J.M. 2006. Exposure to PBDEs and PCDEs associated with the consumption of edible marine species. Environmental Science Technology, 40: 4394-4399.

EASTON, M.D.L; LUSZNIAK D. \& VON DER GEEST, E. 2002. Preliminary examination of contaminant loadings in farmed salmon, wild salmon and commercial salmon feed. Chemosphere, 46(7): 1053-1074.

ENVIRON. 2003. Voluntary children's chemical evaluation program pilot. Tier I assessment of the potential health risks to children associated with exposure to the commercial octabromodiphenyl ether product. CAS No. 32536-52-0. Emeryville, CA: ENVIRON Int. Corp.

EPA (ENVIRONMENTAL PROTECTION AGENCY). 1995. Toxic chemical release inventory. Pp 342-389. Reporting form R and instructions. Washington, DC:U.S. Environmental Protection Agency, Office of Pollution Prevention and Toxics. EPA745K95051. 
ERIKSSON, P.; ANAKARBERG, E. \& VIBERG, H. 2001. Neonatal exposure to toxicants defined critical period: altered adult susceptibility. Neurotoxicology, 22(4): 510.

EU (EUROPEAN UNION). 2002. Bis(pentabromophenyl) ether. Pp 1-279. European Union Risk Assessment Report. Luxembourg: Office for Official Publications of the European Committees.

EU (EUROPEAN UNION). 2003. Diphenyl ether, octabromo derivative. Pp 1-234. European Union Risk Assessment Report. Luxembourg: Office for Official Publications of the European Committees.

FÄNGSTRÖM, B.; ATHANASIADOU, M.; ATHANASSIADIS, I.; BIGNERT, A.; GRANDJEAN, P.; WEIHE, P. \& BERGMAN, Å. 2005. Polybrominated diphenyl ethers and traditional organochlorine pollutants in fulmars (Fulmarus glacialis) from the Faroe Islands. Chemosphere, 60: 836-843.

GREAT LAKES CHEMICAL CORPORATION. 2004. Technical information sheets for DE-60F special, DE-61, DE-62, and DE-71. http://www.el.greatlakes.com, acesso em 19/08/2006.

GUSTAFSSON. K.; BJORK, M. \& BURREAU, S. 1999. Bioaccumulation kinetic of brominated flame retardants (polybrominated diphenyl ethers) in blue mussels (Mytilus edulis). Environmental Toxicolology Chemistry, 18(6): 1218-1224.

HAGLUND, P.S.; ZOOK, D.R.; BUSER, H.R. \& HU, J. 1997. Identification and quantitation of polybrominated diphenyl ethers and methoxy-polybrominated diphenyl ethers in Baltic biota. Environmental Science\& Technology, 31: 3281-3287.

HAGMAR, L.; JAKOBSSON, K. \& THURESSON, K. 2000. Computer technicians are occupationally exposed to polybrominated diphenyl ethers and tetrabromobisphenol. Organohalogen Compounds, 47: 202-205.

HALE, R.C.; LA GUARDIA, M.J. \& HARVEY, E.P. 2002. Potential role of fire retardant-treated polyurethane foam as a source of brominated diphenyl ethers to the US environment. Chemosphere, 46(5): 729-735.

HALE, R.C.; ALAEE, M. \& MANCHESTER-NEESVIG, J.B. 2003. Polybrominated diphenyl ether flame retardants in the North American environment. Environmental Internacional, 29: 771-779.

HALLGREN, S. \& DARNERUD, P. 1998. Effects of polybrominated diphenyl ethers (PBDEs), polychlorinated biphenyls (PCBs) and chlorinated paraffins (CPs) on thyroid hormone levels and enzyme activities in rats. Organohalogen Compounds, 35: 391-394.

HAMERS, T.; KAMSTRA, J.H.; SONNEVELD, E.; MURK, A.J.; KESTER, M.H.A.; ANDERSSON, P.L.; LEGLER, J. \& BROUWER, A. 2006. In vitro profiling of the endocrine disrupting potency of brominated flame retardants. Toxicological Science, 92: 157-173.

HAMILTON, M.C. \& FISHER, T. 2006. Brominated diphenyl ethers in selected foods. 8th Annual Workshop on BFRs in the Environment, Ontario Ministry of the Environment, Toronto, ON, June 27-29, 2006.

HARDELL, L.; LINDSTROM, G.; VAN BAVEL, B.; WINGFORS, H.; SUNDELIN, E. \& LILJEGREN, G., 1998. Concentrations of the flame retardant $2 ; 20 ; 4 ; 40$ tetrabrominated diphenyl ether in human adipose tissue in Swedish persons and the risk for non-Hodgkin's lymphoma. Oncology Research, 10: 429-432.

HARDY, M.L. 2002. A comparison of the properties of the major commercial PBDPO/PBDE product to those of major PBB and PCB products. Chemosphere, 45(5): 717-728.

HARNER, T.; SHOEIB, M.; DIAMOND, M.; IKONOMOU, M. \& STERN, G. 2006. Passive sampler derived air concentrations of PBDEs along an urban-rural transect: Spatial and temporal trends. Chemosphere, 64:262-267.

HARRAD, S. \& HUNTER, S. 2006. Concentrations of polybrominated diphenyl ethers in air and soil on a ruralurban transect across a major UK conurbation. Environment Science \& Technology, 40: 4548-4553.

HAZRATI, S., \& HARRAD, S. 2006. PBDEs and PCBs in indoor air: Levels and factors influencing their concentrations. In: 26th International Symposium on Halogenated Persistent Organic Pollutants.

HE, J.; ROBROCK, K.R. \& ALVAREZ-COHEN, L. 2006. Microbial reductive debromination of polybrominated diphenyl ethers (PBDEs). Environment Science \& Technology, 40: 4429-4434

HERRMANN, T.; LOHMANN, N. \& PAEPKE, O. 2005. Polybrominated diphenyl ethers (PBDEs) in fish, fish oil, fish meal and fish feed samples of various origin. Organohalogen Compounds, 67: 640-643.

HITES, R. A.; FORAN, J. A.; SCHWAGER, S. J.; KNUTH, B. A.; HAMILTON, M. C. \& CARPENTER, D. O. 2004. Global assessment of polybrominated diphenyl ethers in farmed andand wild salmon. Environment Science \& Technology, 38: 4945-4949.

HOOPER, H. \& MCDONALD, T.A. 2000. The PBDEs: an emerging environmental challenge and another reason for breast-milk monitoring programs. Environmental Health Perspectives, 108(5): 387-392.

IKONOMOU, M.G.; KELLEY, B.C. \& STERN, G.A. 2005. Spatial and temporal trends of PBDEs in biota from the Canadian Arctic marine environment. Organohalogen Compounds, 67: 950-953. 
KANNAN, K.; JOHNSON, B.; RAPAPORT, D. \& RODAN, B. 2005. Polybrominated diphenyl ethers and polychlorinated biphenyls in human adipose tissue from New York, U.S.A. Organohalogen Compounds, 67: 1659-1662.

KIM, Y.J.; OSAKO, M. \& SAKAI, S.I. 2006. Leaching characteristics of plybrominated diphenyl ethers (PBDEs) from flame-retardant plastics. Cremosphere, 65: 506-513.

KNUTSEN, H.; BERGSTEN, C.; THOMSEN, C.; SLETTA, A.; BECHER, G.; ALEXANDER, J. \& MELTZER, H.M. 2006. Preliminary assessment of PBDE exposure from food in Norway. Organohalogen Compounds, 67: 1624-1627.

LAW, R.J.; ALLCHIN, C.R.; de BOER, J.; COVACI, A.; HERZKE, D.; LEPOM, P.; MORRIS, S.; TRONCZYNSKI, J. \& de WIT, C.A. 2006. Levels and trends of brominated flame retardants in the Euopean environment. Chemosphere, 64: 187-208.

LI, Q.Q.; LOGANATH, A.; CHONG, Y.S. \& OBBARD, J.P. 2005. Determination and occurrence ofpolybrominated diphenyl ethers in maternal adipose tissue from inhabitants of Singapore. Journal of Chromatography, 819: 253-257.

LOVELL (CENTER FOR SUSTAINABLE PRODUCTION). 2005. An investigation of non-halogen substitutes in electronic enclosure and textile applications. University of Massachusetts Lowell.

McDONALD, T.A. 2002. A perspective on the potential health risks of PBDEs. Chemosphere, 46: 745-755.

McPHERSON, A.; THORPE, B \& BLAKE, A. 2004. Brominated Flame Retardants in Dust on Computers: The Case for Safer Chemicals and Better Computer Design. Computer TakeBack Campaign, http://www.computertakeback.org, acesso em 21/10/2006 as 17:14h.

MARSH, G.; BERGMAN, A.; BLADH, L.G.; GILLNER, M. \& JAKOBSSON,E.1998. Synthesis ofp-hydroxybromodiphenyl ethers and binding to the thyroid receptor. Organohalogen Compound,s 37: 305-308.

MEERTS, I.A.T.M.; VAN ZANDEN, J.J.; LUIJKS, E.A.C.; VAN LEEUWEN-BOL, I.; MARSH, G.; JAKOBSSON, E.; BERGMAN, A.A. \& BROUWER, A. 2000. Potent competitive interactions of some brominated flame retardants and related compounds with human transthyretin in vitro. Toxicological Science, 56: 95-104.

MORREALE de ESCOBAR, G.; OBREGON, M.J. \& ESCOBAR del REY, F. 2000. Clinical perspective: Is neuropsychological development related to maternal hypothyreoidism or to maternal hypothyroxinemia? Journal of Clinical Endocrinology \& Metabolism, 85: 3975-3987.

MUELLER, K.E.; MUELLER-SPITZ, S.R.; HENRY, H.F.; VONDERHEIDE, A.P.; SOMAN, R.S.; KINKLE, B.K.
\& SHANN, J.R. 2006. Fate of pentabrominated diphenyl ethers in soil: abiotic sorption, plant uptake, and the impact of interspecies plant interactions. Environment Science \& Technology, 40: 6662-6667.

NAKARI, T. \& PESSALA, P. 2005. In vitro estrogenicity of polybrominated diphenyl ethers. Organohalogen Compounds, 67: 558-561.

NIERENBERG, D. \& GARCÉS, L. 2004. Produção Animal Industrial - A Próxima Crise Global da Saúde? World Society for the Protection of Animals (WSPA). Pp 1-8. Human Health Summary Portugal.

NOREN, K. \& MEIRONYTE, D. 2000. Certain organochlorine and organobromine contaminants in Swedish human milk in perspective of past 20-30 years. Chemosphere, 40: 1111-1123.

NTP (NATIONAL TOXICOLOGY PROGRAM). 1986. Toxicology andCarcinogenesis Studies of Decabromodiphenyl Oxide (CAS No. 1163-19-5) in F344/N Rats and B6C3F1 Mice (Feed Studies). TR 309. Research Triangle Park.

PENG, J.H; HUANG, C.W; WENG, Y.M \& YAK, H.K. 2007. Determination of plybrominated diphenyl ethers (PBDEs) in fish samples from rivers and estuarines in Taiwan. Chemosphere, 66: 1990-1997.

PETREAS, M.; LI, C.; VISITA, P.; GILL, S.; GILL, M. \& GARCHA, J. 2005. Autoshredder and electronic waste as sources of PBDE exposures in California. Organohalogen Compounds, 67: 1012- 1015.

PETREAS, M. \& OROS, D. 2006. PBDEs in California Waste Streams. Organohalogen Compounds, 68: 499-502.

ROBROCK, K.; HE, J. \& ALVAREZ-COHEN, L. 2005. Degradation of polybrominated diphenyl ethers by anaerobic dehalogenating cultures. Organohalogen Compounds, 67: 594-597.

SCHECTER, A.; PÄPKE, O.; HARRIS, T.R.; TUNG, K.C.; MUSUMBA, A.; OLSON, J. \& BIRNBAUM, L. 2006. Polybrominated diphenyl ether (PBDE) levels in an expanded market basket survey of U.S. food and estimated PBDE dietary intake by age and sex. Environmental Health Perspectives, 114: 1515-1520.

SCHIMID, P.; KOHLER, M.; GUJER, E.; ZENNEG, M. \& LANFRANCHI, M. 2007. Persistent organic pollutants, brominated flame retardants and synthetic musks in fish from remote alpine lakes in Switzerland. Chemosphere, 67(9): S16-S21.

SELLSTRÖM, U.; KIERKKEGAARD, A. \& de WIT, C. A. 1998. Polybrominateddiphenylethersandhexabromocyclododecane in sediment and fish from a Swedish river. Environmental Toxicology and Chemistry, 17(6): 1065- 1072. 
SJODIN, A.; THURESSON, K. \& HAGMAR, L. 1999b. Occupational exposure to polybrominated diphenyl ethers at dismantling of electrons. Ambient air and human serum analysis. Organohalogen Compounds, 43: 447-451.

STRANDBERG, B.; DODDER, N.G.; BASU, I. \& HITES, R.A. 2001. Concentrations and spatial variations of polybrominated diphenyl ethers and other organohalogen compounds in Great Lakes air. Environment Science \& Technology, 35: 1078-1083. STRANDMAN, T.; KOISTINEN, J. \& VARTIAINEN, T. 2000. Polybrominated diphenyl ethers (PBDEs) in placenta and human milk. Organohalogen Compounds, 47: 61-64.

THURESSON, K.; HÖGLUND, P.; HAGMAR, L.; SJÖDIN, A.; BERGMAN, Å. \& JAKOBSSON, K. 2006. Apparent halflives of hepta- to decabrominated diphenyl ethers in human serum as determined in occupationally exposed workers. Environmental Health Perspectives, 114: 176-181.

TLUSTOS, C.; PRATT, I.; MCHUGH, B.; TYRRELL, L.; COOPER, H.; DUFFY, C. \& MCGOVERN, E. 2005a. Investigation into levels of polybrominated diphenyl ethers (PBDEs) and hexabromocyclododecane diastereomers (HCBD) in fishery produce available on the Irish market. Organohalogen Compounds, 67: 636-639.

TLUSTOS, C.; PRATT, I.; WHITE, S.; FERNANDES, A. \& ROSE, M. 2005b. Investigation into levels of PCDD/Fs, PCBs and PBDEs in Irish produce. Organohalogen Compounds, 67: 1474-1477.

VIBERG, H.; FREDRIKSSON, A. \& ERIKSSON, P. 2002. Neonatal exposure to the brominated flame retardant 2,2',4,4',4-pentabromodiphenyl ether causes altered susceptibility if the cholinergic transmitter system in the adult mouse. Toxicological Science, 67: 104-107.

WANG, D.; CAI, Z.; JIANG, G.; LEUNG, A.; WONG, M.H. \& WONG, W.K. 2005. Determination of polybrominated diphenyl ethers in soil and sediment from an electronic waste recycling facility. Chemosphere, 60: 810-816.

WEBSTER, P. 2004. Toxycology: Exposure to Flame Retardants on the Rise. Science, 304: 1730-1730.

WEISS, J.; WALLIN, E.; AXMON, A.; JÖNSSON, B.A.G.; ÅKESSON, H.; JANÁK, K.; HAGMAR, L. \& BERGMAN, A. 2006. Hydroxy-PCBs, PBDEs, and HBCDs in serum from an elderly population of Swedish fishermen's wives and associations with bone density. Environment Science \& Technology, 40: 6282-6289.

WHO (WORLD HEALTH ORGANIZATION). 1994. Environmental Health Criteria 162, Polybrominated Diphenyl Ethers, listed.; World Health Organization, International Programme on Chemical Safety. 